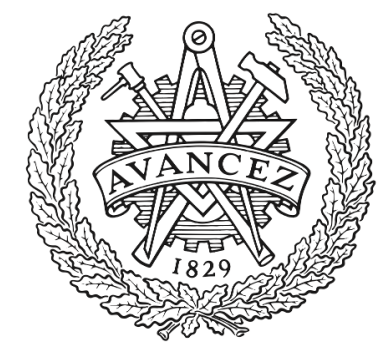

CHALMERS

UNIVERSITY OF TECHNOLOGY

\title{
All Local Gauge Invariants for Perturbations of the Kerr Spacetime
}

Downloaded from: https://research.chalmers.se, 2023-04-26 08:08 UTC

Citation for the original published paper (version of record):

Aksteiner, S., Bäckdahl, T. (2018). All Local Gauge Invariants for Perturbations of the Kerr

Spacetime. Physical Review Letters, 121(5). http://dx.doi.org/10.1103/PhysRevLett.121.051104

N.B. When citing this work, cite the original published paper. 


\title{
All Local Gauge Invariants for Perturbations of the Kerr Spacetime
}

\author{
Steffen Aksteiner ${ }^{1,2, *}$ and Thomas Bäckdahl ${ }^{1,3, \dagger}$ \\ ${ }^{1}$ Albert Einstein Institute, Am Mühlenberg 1, D-14476 Potsdam, Germany \\ ${ }^{2}$ Department of Mathematics, Princeton University, Princeton, 08544-1000 New Jersey, USA \\ ${ }^{3}$ Mathematical Sciences, Chalmers University of Technology and University of Gothenburg, SE-412 96 Gothenburg, Sweden
}

(Received 11 April 2018; published 1 August 2018)

\begin{abstract}
We present two complex scalar gauge invariants for perturbations of the Kerr spacetime defined covariantly in terms of the Killing vectors and the conformal Killing-Yano tensor of the background together with the linearized curvature and its first derivatives. These invariants are, in particular, sensitive to variations of the Kerr parameters. Together with the Teukolsky scalars and the linearized Ricci tensor, they form a minimal set that generates all local gauge invariants. We also present curvature invariants that reduce to the gauge invariants in the linearized theory.
\end{abstract}

DOI: 10.1103/PhysRevLett.121.051104

Introduction.-The black hole perturbation theory plays a major role in numerical and analytical investigations of general relativity. The coordinate freedom or diffeomorphism invariance of the Einstein equations implies gauge freedom for the linearized equations. For many applications, it is essential to extract the gauge-invariant content of the theory. The aim of this Letter is to describe all local gauge invariants for perturbations of the rotating Kerr black hole.

The dynamics of gravitational perturbations of the Schwarzschild geometry is governed by the ReggeWheeler [1] and Zerilli [2] variables (see [3,4] for a gauge-invariant formulation) but also by the BardeenPress [5] variables. Gauge invariants of higher than second differential order have also been used in the literature; see, e.g., Ref. [6] for third-order quantities and Ref. [7] for a set of higher-order gauge invariants and their relations on Schwarzschild. The construction of the Bardeen-Press invariants has been generalized to the Kerr geometry by Teukolsky [8], and Wald showed in Ref. [9] that one complex Teukolsky scalar determines the linearized gravitational field up to unphysical solutions and Kerr parameter variations. Motivated by the self-force problem, Merlin et al. [10] recently constructed three more real, third-order scalar invariants, and in Ref. [11] we found a third-order gauge-invariant vector field. Here we take a different perspective by asking: In terms of which variables can the gauge-invariant content of the theory be described? As will be demonstrated in Ref. [12], there exist a finite number of invariants from which all invariants can be constructed by further differentiation and linear combination. This opens up the possibility of a systematic investigation of the field equations and differential relations satisfied by the gauge-invariant quantities.

Linearized diffeomorphisms generated by a real vector field $\nu^{a}$ change the linearized metric $h_{a b}$ according to

$$
h_{a b} \rightarrow h_{a b}+2 \nabla_{(a} \nu_{b)} .
$$

Depending on the background geometry, certain linear combinations of derivatives of $h_{a b}$ can be constructed to be independent of $\nu^{a}$ under the transformation (1). Such quantities are called local gauge invariants and play a fundamental role in the black hole perturbation theory. Also, nonlocal gauge invariants, often formulated in terms of separated modes or global integrals, can be of interest, but here we restrict our attention to local quantities.

Stewart and Walker [13] showed that any linearized field $\dot{T}$ around a given background transforms under (1) with the Lie derivative along $\nu^{a}$ of its background value $T_{0}$ :

$$
\dot{T} \rightarrow \dot{T}+\mathcal{L}_{\nu} T_{0} .
$$

This, in particular, implies that the linearized Ricci tensor is gauge invariant on vacuum backgrounds.

Any linear differential operator applied to a gauge invariant is also gauge invariant. Therefore, we say that a set of gauge invariants is generating if all gauge invariants can be expressed as a linear combination of differential operators on elements of this set. For instance, the linearized curvature tensor is gauge invariant for perturbations of Minkowski space, and one can show that it forms a generating set.

We would like elements of the generating set to be of as low differential order as possible and also minimal in the sense that the removal of any element implies loss of information about the gauge-invariant content or, equivalently, that the set is no longer generating. Observe, however, that the elements satisfy differential relations. For example, the set of components of the linearized curvature tensor on Minkowski space is minimal generating, but the elements are related by the differential Bianchi identities.

For perturbations of the Kerr spacetime, the two complex Teukolsky scalars (and their derivatives) together with the 
linearized Ricci tensor are well-known gauge invariants. In this Letter, we add two complex scalar fields, $\mathbb{I}_{\xi}$ and $\mathbb{I}_{\zeta}$, to this list of local gauge invariants (collectively, they can be found in Refs. [10,11] — see remarks 5 and 6-but here they are identified as a generating set for the first time). Their construction involves the Killing vectors $\xi^{a}$ and $\zeta^{a}$; see Proposition 1 below. The main result of this Letter is the statement of a minimal generating set of gauge invariants in Theorem 4.

Finally, we present curvature invariants in the nonlinear theory that reduce to constants on a Kerr background and to $\mathbb{I}_{\xi}$ and $\mathbb{I}_{\zeta}$ in the linear theory.

The proof that the set is generating is based on a universal compatibility complex of the Killing operator (see [14]) and will be published separately in Ref. [12]. For examples of the method on Lorentzian manifolds, see, e.g., [15]. The computations for this Letter were performed with XACT for Mathematica, in particular, using the package SPINFRAMES [16].

Geometry of Kerr.-We use abstract index notation and let $g_{a b}$ denote the background Kerr metric with parameters a and M. Unless otherwise stated, frame-dependent statements are valid in any principal Newman-Penrose tetrad $\left(l^{a}, n^{a}, m^{a}, \bar{m}^{a}\right)$ on the background. For clarity, some coordinate expressions are given in Boyer-Lindquist-like coordinates $(t, r, x=\cos \theta, \phi)$.

Let $\mathcal{Y}_{a b}$ be the anti-self-dual conformal Killing-Yano tensor of the Kerr spacetime (see [17]), normalized so that

$$
\xi^{c}=\frac{2}{3} i \nabla_{a} \mathcal{Y}^{c a}
$$

is the real Killing vector $\partial_{t}$. Furthermore, let

$$
p \equiv \sqrt{\mathcal{Y}_{b d} \mathcal{Y}^{b d}}=r-i \mathbf{a} x, \quad U_{a} \equiv-\nabla_{a} \log (p) .
$$

We base our construction on the 2-form $\mathcal{Y}_{a b}$, which in any principal tetrad takes the form

$$
\mathcal{Y}_{a b}=i p\left(l_{[a} n_{b]}-m_{[a} \bar{m}_{b]}\right) .
$$

A second, linearly independent Killing vector is given by

$$
\zeta^{a} \equiv 2 \mathcal{Y}^{a b} \overline{\mathcal{Y}}_{b c} \xi^{c}-\frac{1}{4}\left(p^{2}+\bar{p}^{2}\right) \xi^{a}=\mathbf{a}^{2}\left(\partial_{t}\right)^{a}+\mathbf{a}\left(\partial_{\phi}\right)^{a} ;
$$

see [18] for details. For later reference, we note the reality conditions

$$
\Psi_{2} p^{3}=\bar{\Psi}_{2} \bar{p}^{3}=-\mathbf{M}, \quad \bar{U}^{a} \overline{\mathcal{Y}}_{a b}=-U^{a} \mathcal{Y}_{a b}=\frac{1}{2} i \xi_{b}
$$

and the differential relation

$$
\begin{aligned}
\nabla_{a} U_{b}= & 2 U_{a} U_{b}-\frac{1}{2} g_{a b}\left(\Psi_{2}+2 U_{c} U^{c}\right)-p^{-2} \xi_{a} \xi_{b} \\
& +2 \bar{\Psi}_{2} p^{-2} \mathcal{Y}_{(a}{ }^{c} \overline{\mathcal{Y}}_{b) c} .
\end{aligned}
$$

Metric perturbations.-To avoid complications with the tetrad gauge freedom, we treat metric perturbations covariantly in the style of Ref. [19] and denote the variation of a field $F$ by $\dot{F}$. Define the following version of the linearized Riemann tensor:

$\dot{R}_{a b c d} \equiv 2 g_{f[d} \nabla_{c]} \nabla_{[a} h_{b]}^{f}+\frac{2}{3} R_{[a b]}^{f}{ }_{[c} h_{d] f}-\frac{2}{3} R^{f}{ }_{[a b][c} h_{d] f}$,

which is the mean value of variations with all indices up and all indices down. The spin-0 and spin-1 parts of the linearized curvature can be expressed by

$6 p^{2} \vartheta \Psi_{2}=\left(\dot{R}_{b}{ }^{d}{ }_{c d} \mathcal{Y}_{a}{ }^{c}-\dot{R}_{a b c d} \mathcal{Y}^{c d}-\dot{R}_{a c b d} \mathcal{Y}^{c d}\right) \mathcal{Y}^{a b}$,

$4 p^{2} \mathcal{Z}_{a b}=-2 \mathcal{Y}^{c d} \dot{R}_{[a|c d|}{ }^{f} \mathcal{Y}_{b] f}+3 \mathcal{Y}^{c d} \dot{R}_{[a}{ }^{f}|c d| \mathcal{Y}_{b] f}$.

In any principal tetrad, we get

$$
\begin{aligned}
& \vartheta \Psi_{0}=\dot{R}_{l m l m}, \\
& \vartheta \Psi_{1}=\frac{1}{2} \dot{R}_{l m l n}-\frac{1}{2} \dot{R}_{l m m \bar{m}}, \\
& \vartheta \Psi_{2}=\frac{1}{6} \dot{R}_{l n l n}-\frac{1}{3} \dot{R}_{l n m \bar{m}}+\frac{1}{3} \dot{R}_{l m \bar{m} n}+\frac{1}{6} \dot{R}_{m \bar{m} m \bar{m}}, \\
& \vartheta \Psi_{3}=\frac{1}{2} \dot{R}_{l n \bar{m} n}-\frac{1}{2} \dot{R}_{m \bar{m} \bar{m} n}, \\
& \vartheta \Psi_{4}=\dot{R}_{\bar{m} n \bar{m} n}, \\
& \mathcal{Z}_{a b}=-2 \vartheta \Psi_{3} l_{[a} m_{b]}+2 \vartheta \Psi_{1} \bar{m}_{[a} n_{b]} .
\end{aligned}
$$

Here, $\vartheta \Psi_{i}$ are the components of the linearized Weyl spinor $\vartheta \Psi_{A B C D}$ introduced in Ref. [19], but the formulas above can be used as definitions. They are related to linearized Newman-Penrose Weyl scalars $\dot{\Psi}_{i}$ but compensated for their linearized tetrad gauge dependence:

$$
\begin{aligned}
& \vartheta \Psi_{0}=\dot{\Psi}_{0}, \quad \vartheta \Psi_{1}=\dot{\Psi}_{1}+\frac{3}{2} \Psi_{2}\left(m_{a} \dot{l}^{a}-l_{a} \dot{m}^{a}\right), \quad \vartheta \Psi_{2}=\dot{\Psi}_{2}, \\
& \vartheta \Psi_{4}=\dot{\Psi}_{4}, \quad \vartheta \Psi_{3}=\dot{\Psi}_{3}-\frac{3}{2} \Psi_{2}\left(n_{a} \dot{\bar{m}}^{a}-\bar{m}_{a} \dot{n}^{a}\right) .
\end{aligned}
$$

The set of gauge invariants.-We can now construct third-order gauge invariants. 
Proposition 1.- - Let $V^{a}$ be a real Killing vector field and

$$
\begin{aligned}
\mathbb{I}_{V}= & p^{2} W^{a} \nabla_{a}\left(p^{4} \vartheta \Psi_{2}\right)-\frac{1}{2} \operatorname{Re}\left(p^{6} \vartheta \Psi_{2} \nabla_{a} W^{a}\right) \\
& -2 i \operatorname{Im}\left(p^{6} U^{a} W^{b} \mathcal{Z}_{a b}\right)-\frac{3}{2} p^{6} \Psi_{2} U^{a} W^{b} h_{a b},
\end{aligned}
$$

where the vector field $W_{a} \equiv 2 i p^{-3} V^{b} \mathcal{Y}_{a b}$ is assumed to satisfy the condition

$$
\bar{p}^{3} \bar{U}_{[a} \bar{W}_{b]}=-p^{3} U_{[a} W_{b]} .
$$

Then $\mathbb{I}_{V}$ is a local gauge invariant.

Proof.-A consequence of the Killing equation gives $\Psi_{2} U^{a} V_{a}=0$. For a pure diffeomorphism, we get

$$
\begin{aligned}
\vartheta \Psi_{2}= & 3 \Psi_{2} U^{a} \nu_{a}, \\
\mathcal{Y}_{b}{ }^{c} \mathcal{Z}_{a c}= & -\frac{3}{2} \Psi_{2} \mathcal{Y}_{b}{ }^{c} \nabla_{[a} \nu_{c]}+\frac{3}{2} \Psi_{2} \mathcal{Y}_{a}{ }^{c} \nabla_{[b} \nu_{c]} \\
& -3 \Psi_{2} U_{[a} \mathcal{Y}_{b]}{ }^{c} \nu_{c}-3 \Psi_{2} U^{c} \mathcal{Y}_{[a|c|} \nu_{b]} .
\end{aligned}
$$

This gives

$$
\begin{aligned}
p^{6} U^{a} W^{b} \mathcal{Z}_{a b}= & -\frac{3}{2} p^{6} \Psi_{2} U^{a} W^{b}\left(\nabla_{[a} \nu_{b]}+2 U_{[a} \nu_{b]}\right) \\
& -3 i p^{3} \Psi_{2} U^{a} V^{b} \mathcal{Y}_{a}{ }^{c} \nabla_{[b} \nu_{c]} .
\end{aligned}
$$

The conditions (7) and (14) then imply

$$
\begin{aligned}
i \operatorname{Im} & \left(p^{6} U^{a} W^{b} \mathcal{Z}_{a b}\right) \\
& =-\frac{3}{2} \Psi_{2} U^{a} W^{b} p^{6}\left(\nabla_{[a} \nu_{b]}+U_{[a} \nu_{b]}+\bar{U}_{[a} \nu_{b]}\right) .
\end{aligned}
$$

Together with (15a), we get for a pure diffeomorphism

$$
\begin{aligned}
\mathbb{I}_{V}= & -3 \Psi_{2} U^{a} U^{b} W_{a} p^{6} \nu_{b}-\frac{3}{4} \Psi_{2} U^{a} p^{6} \nu_{a} \nabla_{b} W^{b} \\
& -\frac{3}{4} \bar{\Psi}_{2} \bar{U}^{a} \bar{p}^{6} \nu_{a} \nabla_{b} \bar{W}^{b}+3 \Psi_{2} W^{a} p^{6} \nu^{b} \nabla_{(a} U_{b)} \\
& +3 \Psi_{2} U^{a} W^{b} p^{6}\left(U_{[a} \nu_{b]}+\bar{U}_{[a} \nu_{b]}\right) .
\end{aligned}
$$

The relations (7), (8), and (14) imply

$$
\begin{aligned}
W^{b} \nabla_{b} U_{a}= & 2 U_{a} U^{b} W_{b}-U_{b} U^{b} W_{a}-\Psi_{2} \operatorname{Re}\left(W_{a}\right), \\
U^{b} \nabla_{b} W_{a}= & 2 U^{b} U_{(a} W_{b)}-\Psi_{2} \operatorname{Re}\left(W_{a}\right), \\
\bar{p}^{3} \bar{U}_{a} \nabla_{b} \bar{W}^{b}= & -4 \Psi_{2} \operatorname{Re}\left(W_{a}\right) p^{3}-U_{a} p^{3} \nabla_{b} W^{b} \\
& +4\left(U^{b}-\bar{U}^{b}\right) p^{3} U_{[a} W_{b]} .
\end{aligned}
$$

Together, we get $\mathbb{I}_{V}=0$ for a pure diffeomorphism.

Remark 2.-For the case $V^{a}=\xi^{a}$ we get $W^{a}=-U^{a} p^{-1}$, and for the case $V^{a}=\zeta^{a}$ we get

$$
W_{a} p^{3}=-\frac{1}{2} \bar{U}_{a} p^{2} \bar{p}^{2}+\frac{1}{4} U_{a} p^{2}\left(p^{2}+\bar{p}^{2}\right) .
$$

Both of these vectors satisfy the required condition (14), so $\mathbb{I}_{\xi}$ and $\mathbb{I}_{\zeta}$ are gauge invariant.

Corollary 3.-A set of local gauge-invariant quantities for perturbations of the Kerr spacetime is given by

$$
\begin{array}{ll}
\text { Teukolsky scalars } & \vartheta \Psi_{0}, \vartheta \Psi_{4}, \\
\text { linearized Ricci } & \dot{R}_{a b}=\dot{R}_{a c b}{ }^{c}, \\
\text { Killing invariants } & \mathbb{I}_{\xi}, \mathbb{I}_{\zeta} .
\end{array}
$$

Note that (21a) and (21b) depend on up to second derivatives of linearized metric, while (21c) depends on up to third derivatives.

Theorem 4 ([12]).- The set of gauge invariants in Corollary 3 is minimal and generates all local gauge invariants for perturbations of the Kerr spacetime with $\mathbf{a} \neq 0$.

Arguments for minimality are given below, and for a proof of the theorem we refer to Ref. [12].

It should be noted that a generating set of gauge invariants can degenerate if restricted to more special backgrounds, in the sense that certain components of the set can be derived from more elementary gauge invariants. Examples are the second-order Regge-Wheeler variable on Schwarzschild and the linearized curvature components of spin 0 and 1 on Minkowski.

Also, the spherical Killing vectors on Schwarzschild satisfy condition (14) and therefore lead to gauge invariants. On the other hand, the Regge-Wheeler variable $\operatorname{Im} \vartheta \Psi_{2}$ is gauge invariant, and hence certain real or imaginary parts of Killing invariants can be generated from it.

Remark 5.--In Ref. [11], we derived a covariant version of the Teukolsky-Starobinski identities. In these identities, a real, gauge-invariant vector field $\operatorname{Im} \mathcal{A}^{c}$ appears naturally. It can be expressed in terms of the invariants (21); for example, $\operatorname{Im} \mathcal{A}^{a} V_{a}=-\frac{1}{81} \operatorname{Im} \mathbb{I}_{V}$ for both isometries in the source-free case. This partly initiated the systematic search for gauge invariants.

Remark 6.-Merlin et al. found three real gauge invariants [10] in a coordinate-based construction. They are related to $\operatorname{ReI}_{\xi}, \operatorname{ReI}_{\zeta}$, and $\operatorname{ImI}_{\xi}$ via

$$
\begin{aligned}
& \mathcal{I}_{1}=-\frac{2\left[\operatorname{Re}\left(\mathbb{I}_{\zeta}\right)+r^{2} \operatorname{Re}\left(\mathbb{I}_{\xi}\right)\right]\left(r^{2}+\mathbf{a}^{2} x^{2}\right)}{3 \mathbf{M}\left(r^{2}-2 \mathbf{M} r+\mathbf{a}^{2}\right)^{2}}, \\
& \mathcal{I}_{2}=-\frac{2\left[\operatorname{Re}\left(\mathbb{I}_{\zeta}\right)-\mathbf{a}^{2} x^{2} \operatorname{Re}\left(\mathbb{I}_{\xi}\right)\right]\left(r^{2}+\mathbf{a}^{2} x^{2}\right)}{3 \mathbf{M a}^{2}\left(1-x^{2}\right)}, \\
& \mathcal{I}_{3}=\frac{\operatorname{Im}\left(\mathbb{I}_{\xi}\right)\left(r^{2}+\mathbf{a}^{2} x^{2}\right)^{2}}{3 \mathbf{M a}\left(r^{2}-2 \mathbf{M} r+\mathbf{a}^{2}\right) \sqrt{1-x^{2}}} .
\end{aligned}
$$


Type $D$ variations and independence of gauge invariants.-We use the Plebanski-Demianski solution [20] in a vacuum. In coordinates $(t, r, x=\cos \theta, \phi)$, define the Newman-Penrose tetrad

$$
\begin{aligned}
l & =\frac{(1-\mathbf{c} r x)}{\sqrt{2 \Sigma \Delta_{r}}}\left[\left(r^{2}+\mathbf{a}^{2}\right) \partial_{t}+\Delta_{r} \partial_{r}+\mathbf{a} \partial_{\phi}\right], \\
n & =\frac{(1-\mathbf{c} r x)}{\sqrt{2 \Sigma \Delta_{r}}}\left[\left(r^{2}+\mathbf{a}^{2}\right) \partial_{t}-\Delta_{r} \partial_{r}+\mathbf{a} \partial_{\phi}\right], \\
m & =\frac{(1-\mathbf{c} r x)}{\sqrt{2 \Sigma \Delta_{x}}}\left[i \mathbf{a}\left(1-x^{2}\right) \partial_{t}-\Delta_{x} \partial_{x}+i \partial_{\phi}\right], \\
\Delta_{x} & =1+2 \mathbf{N a}^{-1} x-x^{2}+2 \mathbf{c M} x^{3}-\mathbf{c}^{2} a^{2} x^{4}, \\
\Delta_{r} & =\mathbf{a}^{2}-2 \mathbf{M} r+r^{2}-2 \mathbf{c N a} \mathbf{a}^{-1} r^{3}-\mathbf{c}^{2} r^{4}, \\
\Sigma & =r^{2}+\mathbf{a}^{2} x^{2},
\end{aligned}
$$

with parameters $\mathbf{M}, \mathbf{N}, \mathbf{a}$, and $\mathbf{c}$ for mass, Newman-UntiTamburino (NUT) charge, angular momentum, and $c$ metric, respectively. A variation in each of the parameters leads to specific values of the invariants showing their functional independence.

For pure mass $(\dot{\mathbf{M}})$ and angular momentum $(\dot{\mathbf{a}})$ perturbations, the invariants take the form

$$
\mathbb{I}_{\xi}=\dot{\mathbf{M}}, \quad \mathbb{I}_{\zeta}=2 \mathbf{a}^{2} \dot{\mathbf{M}}-3 \mathbf{M a} \dot{\mathbf{a}},
$$

while perturbing in the direction of the NUT $(\dot{\mathbf{N}})$ yields

$$
\begin{aligned}
& \mathbb{I}_{\xi}=-i \dot{\mathbf{N}}+\frac{2 i \mathbf{M}}{\bar{p}} \dot{\mathbf{N}}, \\
& \mathbb{I}_{\zeta}=-i \mathbf{a}^{2} \dot{\mathbf{N}}+\mathbf{a} x\left(r-2 \mathbf{M}-\frac{\mathbf{M} p}{\bar{p}}\right) \dot{\mathbf{N}},
\end{aligned}
$$

and perturbing in the $c$-metric direction $(\dot{\mathbf{c}})$ gives

$$
\begin{aligned}
& \mathbb{I}_{\xi}=\frac{6 \mathbf{M}^{2} r x}{\bar{p}} \dot{\mathbf{c}}+3 \mathbf{M}[i \mathbf{a}+(\mathbf{M}-r) x] \dot{\mathbf{c}}, \\
& \mathbb{I}_{\zeta}=\frac{6 \mathbf{M}^{2} \mathbf{a}^{2} r x^{3}}{\bar{p}} \dot{\mathbf{c}}-3 i \mathbf{M a}\left(p^{2}-r^{2} x^{2}\right) \dot{\mathbf{c}} .
\end{aligned}
$$

Observe that $\mathbb{I}_{\xi}$ and $\mathbb{I}_{\zeta}$ are real for $\dot{\mathbf{M}}$ and $\dot{\mathbf{a}}$ perturbations but complex for $\dot{\mathbf{N}}$ and $\dot{\mathbf{c}}$ perturbations. From the explicit form above, we conclude that the four real degrees of freedom of $\mathbb{I}_{\xi}$ and $\mathbb{I}_{\zeta}$ are functionally independent. Furthermore, there are algebraically special frequency solutions (see, e.g., [21]), turning on only one of $\vartheta \Psi_{0}$ and $\vartheta \Psi_{4}$. Similarly, metric perturbations turning on specific components of the linearized Ricci tensor are possible to construct by a linearized conformal transformation. Hence, we have a sequence of solutions turning on one invariant after the other. This motivates why all 18 invariants are needed on Kerr with $\mathbf{a} \neq 0$. Even though the gauge invariants are independent in this way, they will satisfy a set of differential compatibility equations. These relations will be stated and used in [12] for the proof of Theorem 4.

One can argue that components of the linearized curvature are the only possible gauge invariants of second order and that no gauge-invariant curvature component carries the $\dot{\mathbf{M}}, \dot{\mathbf{a}}, \dot{\mathbf{N}}$, and $\dot{\mathbf{c}}$ perturbations. The differential order of $\mathbb{I}_{\xi}$ and $\mathbb{I}_{\zeta}$ is therefore minimal.

Geroch-Held-Penrose (GHP) form of gauge invariants. - In a principal tetrad, the Killing invariants take the GHP [22] form

$$
\begin{aligned}
& \mathbb{I}_{\xi}=-p\left(\rho^{\prime} \mathbf{P}+\rho \mathbf{P}^{\prime}-\tau^{\prime} ð-\tau ð^{\prime}\right)\left(p^{4} \vartheta \Psi_{2}\right)-\frac{1}{2} \Psi_{2} p^{5} \vartheta \Psi_{2} \\
& -\frac{1}{2} \bar{\Psi}_{2} \bar{p}^{5} \overline{\vartheta \Psi}_{2}+\frac{3}{2} \Psi_{2} p^{5}\left(h_{n n} \rho^{2}+2 h_{l n} \rho \rho^{\prime}+h_{l l} \rho^{\prime 2}\right. \\
& -2 h_{n \bar{m}} \rho \tau-2 h_{l \bar{m}} \rho^{\prime} \tau+h_{\bar{m} \bar{m}} \tau^{2}-2 h_{n m} \rho \tau^{\prime} \\
& \left.-2 h_{l m} \rho^{\prime} \tau^{\prime}+2 h_{m \bar{m}} \tau \tau^{\prime}+h_{m m} \tau^{\prime 2}\right)
\end{aligned}
$$

and, with $p_{+}=p+\bar{p}, p_{-}=p-\bar{p}$,

$$
\begin{aligned}
\mathbb{I}_{\zeta}= & \frac{1}{4} p\left[p_{-}^{2}\left(\rho^{\prime} \mathbf{P}+\rho \mathbf{P}^{\prime}\right)-p_{+}^{2}\left(\tau^{\prime} \text { ठ }+\tau \nwarrow^{\prime}\right)\right]\left(p^{4} \vartheta \Psi_{2}\right) \\
& +\frac{1}{4} \operatorname{Re}\left\{p^{5} \vartheta \Psi_{2}\left[\Psi_{2} p_{+} p_{-}-2 \bar{\Psi}_{2} \bar{p}^{2}-4 p\left(p_{-} \rho \rho^{\prime}-p_{+} \tau \tau^{\prime}\right)\right]\right\} \\
& +2 i \operatorname{Im}\left[p^{6} \bar{p}\left(\vartheta \Psi_{3} \rho \tau+\vartheta \Psi_{1} \rho^{\prime} \tau^{\prime}\right)\right] \\
& -\frac{3}{8} \Psi_{2} p^{5}\left[p_{-}^{2}\left(h_{n n} \rho^{2}+2 h_{l n} \rho \rho^{\prime}+h_{l l} \rho^{\prime 2}\right)\right. \\
& -2 p_{+} p_{-}\left(h_{n \bar{m}} \rho \tau+h_{l \bar{m}} \rho^{\prime} \tau+h_{n m} \rho \tau^{\prime}+h_{l m} \rho^{\prime} \tau^{\prime}\right) \\
& \left.+p_{+}^{2}\left(h_{\bar{m} \bar{m}} \tau^{2}+2 h_{m \bar{m}} \tau \tau^{\prime}+h_{m m} \tau^{\prime 2}\right)\right] .
\end{aligned}
$$

Curvature invariants.-The scalars $\mathbb{I}_{\xi}$ and $\mathbb{I}_{\zeta}$ can be derived from linearizations of tensors built from the curvature and its derivatives in the full theory. On a general vacuum spacetime with anti-self-dual Weyl curvature $\mathcal{C}_{a b c d}=\frac{1}{2} C_{a b c d}+\frac{1}{2} i^{*} C_{a b c d}$, define the curvature invariants

$$
\hat{\mathcal{I}}=\frac{1}{24} \mathcal{C}_{a b c d} \mathcal{C}^{a b c d}, \quad \mathcal{I}=\hat{\mathcal{I}}^{1 / 6}
$$

Furthermore, define the complex curvature invariant

$$
\mathbb{M}=-\mathcal{I}^{-4}\left(\nabla_{a} \mathcal{I}\right)\left(\nabla^{a} \mathcal{I}\right)+\mathcal{I}+\overline{\mathcal{I}}
$$

On a Kerr spacetime in a principal tetrad, we find $\hat{\mathcal{I}}=\Psi_{2}^{2}$, and $\mathbb{M}$ turns out to be the real constant $\mathbb{M}_{\text {Kerr }}=\mathbf{M}^{-2 / 3}$. Because of (2), it follows that the variation of (28) around Kerr is gauge invariant, and a lengthy calculation shows 


$$
\dot{\mathbf{M}}=-\frac{2}{3} \mathbf{M}^{-5 / 3} \mathbb{I}_{\xi}
$$

Similarly define the real scalar curvature invariant

$$
\mathbb{A}=\frac{-1}{|\mathcal{I}|^{4}}\left(\nabla_{a} \frac{\mathcal{I}}{\overline{\mathcal{I}}}\right)\left(\nabla^{a} \frac{\overline{\mathcal{I}}}{\overline{\mathcal{I}}}\right)-2 \operatorname{Im}\left(\frac{1}{\mathcal{I}^{2}}\right) \operatorname{Im}(2 \mathcal{I}-\mathbb{M}) .
$$

In the background, it reduces to $A_{\text {Kerr }}=4 \mathbf{a}^{2} \mathbf{M}^{-4 / 3}$. The variation of $\mathbb{A}$ around a Kerr background shows

$$
\dot{A}=-\frac{8}{3} \mathbf{M}^{-7 / 3} \operatorname{ReI}_{\zeta} .
$$

To express $\mathrm{ImI}_{\zeta}$, define the real, symmetric, trace-free two tensor

$$
T_{a c}=\operatorname{Im}\left[\mathcal{I}^{8} \overline{\mathcal{I}}^{5} \overline{\mathcal{C}}_{\left(a^{b}{ }^{b}\right)}{ }^{d}\left(\nabla_{b} \mathcal{I}\right)\left(\nabla_{d} \overline{\mathcal{I}}\right)\right],
$$

which equals Eq. (18) in Ref. [23] up to a constant. On a general type $D$ spacetime, it has the nontrivial factor $\bar{\rho} \tau+\rho \bar{\tau}^{\prime}$, which is zero in the Kerr case. Variation around Kerr and contraction into $\xi^{a}$ lead to

$$
\dot{T}_{a c} \xi^{a} \xi^{c}=\frac{\Psi_{2}^{5} p^{4}}{\bar{p}^{11}}\left(\xi^{c} \zeta_{c} \operatorname{ImI}_{\xi}-\xi^{c} \xi_{c} \operatorname{Im} \mathbb{I}_{\zeta}\right) .
$$

Conclusions. - In this Letter, we introduced two complex scalar gauge invariants for perturbations of Kerr spacetime. Together with the Teukolsky scalars and the Ricci tensor, they form a minimal generating set of 18 real scalar invariants. A similar construction on a Schwarzschild background leads to a set of 19 real scalar invariants, and for Minkowski space it is known to consist of the 20 real scalar components of the linearized curvature tensor; see, e.g., [15]. Whether there is a relation between the minimal number of generators for gauge invariants and the number of parameters of the background, also in other spacetimes, is yet unclear.

We would also like to point out that the invariants as defined in (13) directly depend on the background isometries. The alternative definition in terms of curvature invariants does not make explicit use of this structure and may be interesting for tracking type $D$ parameter variations in numerical evolution as well as for higher-order self-force problems. It would also be interesting to analyze the set of gauge invariants from the perspective of the black hole stability problem. Assuming that the Teukolsky scalars are under control, relations to the other invariants can be analyzed without gauge fixing and yield additional flexibility for the integration of the remaining field equations after gauge fixing.

The geometric background and the full proof of Theorem 4 will be given in Ref. [12].

We thank B. Whiting, L. Andersson, and I. Khavkine for many enlightening discussions and, in particular, B. Whiting for bringing the problem of finding additional gauge invariants to our attention. We thank $M$. van de Meent for pointing out Ref. [10] and for discussions. S. A. thanks Princeton University for hospitality and financial support.

*steffen.aksteiner@aei.mpg.de

†thomas.backdahl@aei.mpg.de

[1] T. Regge and J. A. Wheeler, Stability of a Schwarzschild singularity, Phys. Rev. 108, 1063 (1957).

[2] F. J. Zerilli, Effective Potential for Even-Parity ReggeWheeler Gravitational Perturbation Equations, Phys. Rev. Lett. 24, 737 (1970).

[3] V. Moncrief, Gravitational perturbations of spherically symmetric systems. I. The exterior problem, Ann. Phys. (N.Y.) 88, 323 (1974).

[4] J. Jezierski, Energy and angular momentum of the weak gravitational waves on the Schwarzschild backgroundquasilocal gauge-invariant formulation, Gen. Relativ. Gravit. 31, 1855 (1999).

[5] J. M. Bardeen and W. H. Press, Radiation fields in the Schwarzschild background, J. Math. Phys. (N.Y.) 14, 7 (1973).

[6] G. Dotti, Nonmodal Linear Stability of the Schwarzschild Black Hole, Phys. Rev. Lett. 112, 191101 (2014).

[7] A. G. Shah, B. F. Whiting, S. Aksteiner, L. Andersson, and T. Bäckdahl, Gauge-invariant perturbations of Schwarzschild spacetime, arXiv:1611.08291.

[8] S. A. Teukolsky, Perturbations of a rotating black hole. I. Fundamental equations for gravitational, electromagnetic, and neutrino-field perturbations, Astrophys. J. 185, 635 (1973).

[9] R. M. Wald, On perturbations of a Kerr black hole, J. Math. Phys. (N.Y.) 14, 1453 (1973).

[10] C. Merlin, A. Ori, L. Barack, A. Pound, and M. van de Meent, Completion of metric reconstruction for a particle orbiting a Kerr black hole, Phys. Rev. D 94, 104066 (2016).

[11] S. Aksteiner, L. Andersson, and T. Bäckdahl, New identities for linearized gravity on the Kerr spacetime, arXiv: 1601.06084.

[12] S. Aksteiner, L. Andersson, T. Bäckdahl, I. Khavkine, and B. Whiting, Compatibility complex for black hole spacetimes (to be published).

[13] J. M. Stewart and M. Walker, Perturbations of spacetimes in general relativity, Proc. R. Soc. A 341, 49 (1974).

[14] I. Khavkine, Compatibility complexes of overdetermined PDEs of finite type, with applications to the Killing equation, arXiv:1805.03751.

[15] I. Khavkine, The Calabi complex and Killing sheaf cohomology, J. Geom. Phys. 113, 131 (2017).

[16] S. Aksteiner and T. Bäckdahl, SpinFrames, http://xact.es/ SpinFrames/.

[17] M. Walker and R. Penrose, On quadratic first integrals of the geodesic equations for type $\{2,2\}$ spacetimes, Commun. Math. Phys. 18, 265 (1970). 
[18] C. D. Collinson and P. N. Smith, A comment on the symmetries of Kerr black holes, Commun. Math. Phys. 56, 277 (1977).

[19] T. Bäckdahl and J. A. Valiente Kroon, A formalism for the calculus of variations with spinors, J. Math. Phys. (N.Y.) 57, 022502 (2016).

[20] J. F. Plebanski and M. Demianski, Rotating, charged, and uniformly accelerating mass in general relativity, Ann. Phys. (N.Y.) 98, 98 (1976).
[21] S. Chandrasekhar, The Mathematical Theory of Black Holes, Int. Ser. Monogr. Phys. Vol. 69 (Clarendon, Oxford University Press, New York, 1992).

[22] R. Geroch, A. Held, and R. Penrose, A space-time calculus based on pairs of null directions, J. Math. Phys. (N.Y.) 14, 874 (1973).

[23] J. J. Ferrando and J. A. Sáez, An intrinsic characterization of the Kerr metric, Classical Quantum Gravity 26, 075013 (2009). 